\title{
Modelling of carbon nanotube functionalization processes
}

\author{
Tatyana Dyachkova ${ }^{1, *}$, Evgeny Tugolukov ${ }^{1}$, and Elena Burakova ${ }^{1}$ \\ ${ }^{1}$ Tambov State Technical University, 392000, Tambov, Russia
}

\begin{abstract}
In the present paper, kinetic regularities of oxidative functionalization of carbon nanotubes in nitric acid vapors are studied. The catalytic effect of transition metal oxide particles on the formation of carboxyl groups is shown. Based on the information about changes in the degree of functionalization and composition of gaseous reaction products, the effective values of the thermal effect of the implemented process are determined. A model of the temperature field of the reaction zone of the gas-phase oxidative functionalization of carbon nanotubes process is proposed, thereby making it possible to determine the structural and mode parameters of the equipment.
\end{abstract}

\section{Introduction}

Carbon nanotubes (CNTs) represent a whole class of materials that differ in length, diameter, number and shape of graphene layers, type of electrical conductivity, and volumetric morphology [1]. To date, methods allowing for obtaining the CNTs with specified parameters, including those on an industrial scale, have been developed [2]. Due to their unique mechanical and electrical properties, the CNTs often show high efficiency when used in composites with increased strength characteristics, thermal stability, resistance to hydrothermal aging [3-4], etc. However, when the CNTs with high free surface energy are introduced into polymers and solvents, they are prone to agglomeration phenomena, because of which the achieved effects on improving the properties of modifiable matrices are significantly reduced. Most often, to solve this kind of problem, the CNTs are subjected to various types of chemical treatment in order to form functional groups of different nature for improving dispersibility in polymers and solvents.

A number of papers show the advantages of functionalization by means of gas-phase treatment regarding the consumption of reagents, increase in the achieved indexes of the degree of functionalization, preservation of the structural integrity of surface graphene layers, volume morphology of materials and inclusions of transition metal particles in their composition [5]. The CNTs exposed to the gas-phase functionalization are used in composites with high electrical conductivity and ability to absorb electromagnetic radiation [6].

However, when scaling the processes of chemical treatment of the CNTs for mass production of their functionalized forms, it is necessary to develop conditions in working devices that ensure the production of materials of specified quality.

Considering the aforementioned, the aim of the present research was to experimentally study the kinetic regularities of the CNT gas-phase oxidative functionalization and mathematical modeling of the temperature field in the reaction zone of a device for implementing this process.

\section{Materials and Methods}

In experimental studies, "Taunit-M" cylindrical multiwalled CNTs $\left(\mathrm{d}=8 \div 15 \mathrm{~nm}, 1 \geq 2 \mu \mathrm{m}, \mathrm{S} \geq 300 \mathrm{~m}^{2} / \mathrm{g}\right)$ synthesized at NanoTechCenter Ltd. (Tambov, Russia) via the CVD method over a Co/Mo catalyst were used (Fig. 1) . They were purified from the catalyst impurities using a hydrochloric acid solution, and then washed and dried.

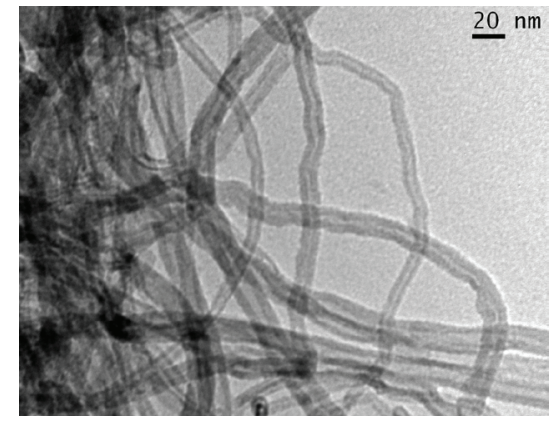

Fig. 1. TEM image of multiwalled CNTs "Taunit-M"

The oxidation in $65 \%$ nitric acid vapors was carried out at a temperature of $140{ }^{\circ} \mathrm{C}$ in a laboratory setup (Fig. 2), which allows for a dosed supply of an oxidizing reagent $(1,2)$ and an inert gas $(3,4,5)$, evaporation of the oxidant , regulation and maintenance of temperature, sampling and measurement of gaseous product volume. The process was performed in a quartz glass tubular reactor consisting of two chambers, one of which was of the vaporizing type (6) for the oxidizing reagent, and the other was for introducing the CNTs (7). The reactor was placed in a tubular resistive electric furnace (8). To control the

\footnotetext{
* Corresponding author: dyachkova tp@mail.ru
} 
temperature, a chromel-alumel thermocouple (9) was used, whereas a microprocessor PID controller (10) was employed to fix the reagent supply. During the experiment, the volume was measured with a rotameter (11), and gaseous reaction products were sampled (12).lower case. You should leave $35 \mathrm{~mm}$ of space above the title and $6 \mathrm{~mm}$ after the title.

Contents of $\mathrm{O}_{2}, \mathrm{~N}_{2}, \mathrm{Ar}$, carbon oxides $\left(\mathrm{CO}, \mathrm{CO}_{2}\right)$ and nitrogen oxides $\left(\mathrm{N}_{2} \mathrm{O}, \mathrm{NO}, \mathrm{NO}_{2}\right)$ in the products were determined by a "Crystal - 2000M" gas chromatograph (GC). Two packed columns filled with zeolite CaA (1 = 2 $\mathrm{m}, \mathrm{d}=3 \mathrm{~mm}$ ) and a "Chromosorb 102" polymeric sorbent ( $l=1 \mathrm{~m}, d=3 \mathrm{~mm}$ ) were used for separation. Concentrations of the components were quantified using a thermal conductivity detector (TCD). Helium was used as carrier gas.

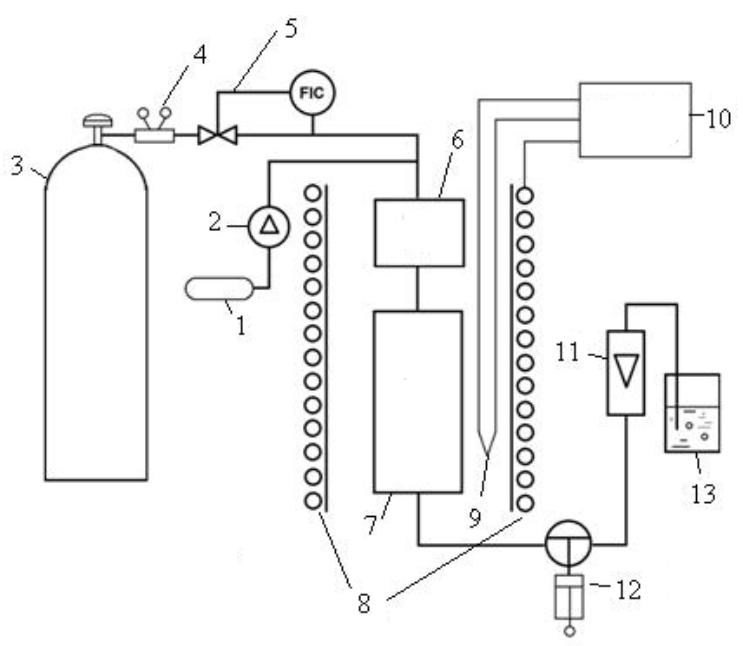

Fig. 2. A scheme of the laboratory setup to study the gas-phase functionalization of carbon nanotubes: 1 - reservoir with an oxidizing reagent; 2 - dispensing pump; 3 -inert gas-containing vessel; 4 - pressure regulator; 5- flow regulator; 6 - vaporizing-type chamber; 7 - reaction chamber; 8 - resistive furnace; 9 - thermocouple; 10- microprocessor temperature controller; 11 - rotameter; 12-gas sampler; 13 - water seal

The degree of the CNT functionalization using the carboxyl groups $\left(D_{f}, \mathrm{mmol} / \mathrm{g}\right)$ was estimated according to the titrimetric method described elsewhere [7].

\section{Experimental Results}

The change in the degree of functionalization under the oxidation in the nitric acid vapors is shown in Fig. 3. During the process, the dependence of the $D_{f}$ on the duration of the treatment of the previously purified CNTs reaches a certain limiting value corresponding to the saturation of their surface with the functional groups. The impurities (admixtures) of transition metal particles in the initial CNTs have a catalyzing effect on the formation of the functional groups. In the investigated interval of time, the $D_{f}$ limiting value is not achieved.

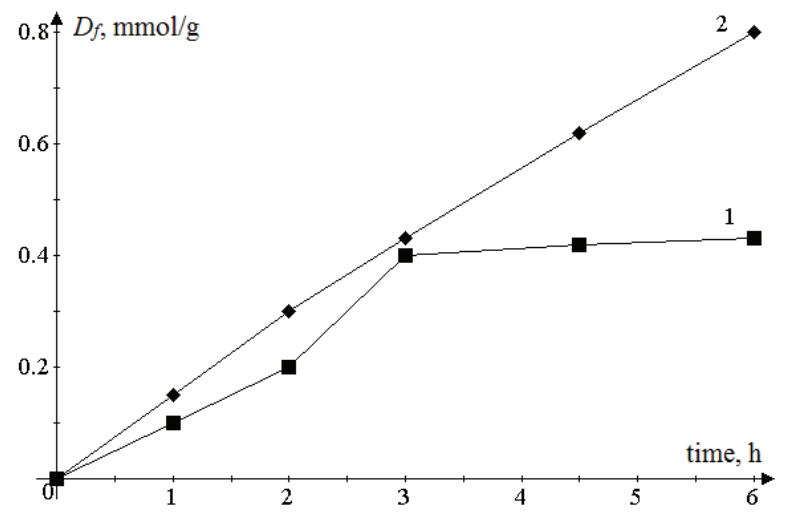

Fig. 3. The change in the degree of the functionalization with carboxyl groups during oxidation in nitric acid vapors at $140^{\circ} \mathrm{C}$ for the CNTs previously purified from the metal oxide catalyst admixtures (1) and the initial CNTs (2)

The composition of the gaseous products of the CNT interaction with the nitric acid vapor after water vapor condensation includes oxides of nitrogen and carbon (Table 1).

Table 1. Volume $(V)$ and composition of gaseous oxidation products of $1 \mathrm{~g}$ of the CNTs in nitric acid vapors at $140^{\circ} \mathrm{C}$ according to gas chromatographic analysis data

\begin{tabular}{|c|c|l|l|l|l|l|}
\hline \multirow{3}{*}{$\begin{array}{c}\text { CNT } \\
\text { type }\end{array}$} & \multirow{2}{*}{$\begin{array}{c}\text { Time, } \\
\mathrm{h}\end{array}$} & $\begin{array}{c}V \\
\text { (norm. } \\
\text { cond.), } \\
\end{array}$ & & \multicolumn{4}{|c|}{$\begin{array}{c}\text { Component proportion in } \\
\text { gas mixture, v. \% }\end{array}$} \\
\cline { 4 - 8 } & & $\mathrm{CO}$ & $\mathrm{CO}_{2}$ & $\mathrm{~N}_{2} \mathrm{O}$ & $\mathrm{NO}_{2}$ \\
\hline \multirow{5}{*}{ initial } & 1.5 & 0.78 & 12.2 & 5.3 & 0 & 82.5 \\
\cline { 2 - 7 } & 2.5 & 1.51 & 6.8 & 21.5 & 0.5 & 71.2 \\
\cline { 2 - 8 } & 3.5 & 2.01 & 6.8 & 17.7 & 0.6 & 74.9 \\
\cline { 2 - 7 } & 4.5 & 2.53 & 6.0 & 16.7 & 0.5 & 76.8 \\
\cline { 2 - 7 } & 6.0 & 3.17 & 5.1 & 13.6 & 0.9 & 80.4 \\
\hline \multirow{5}{*}{ purified } & 2.5 & 1.43 & 6.1 & 17.1 & 1.7 & 75.1 \\
\cline { 2 - 7 } & 3.5 & 1.95 & 5.7 & 15.8 & 1.2 & 77.3 \\
\cline { 2 - 7 } & 4.5 & 2.51 & 5.5 & 15.7 & 1.4 & 77.4 \\
\cline { 2 - 7 } & 6.0 & 3.11 & 4.8 & 12.8 & 0.9 & 81.5 \\
\hline
\end{tabular}

Nitrogen dioxide formed by the reaction

$$
4 \mathrm{HNO}_{3} \rightarrow 4 \mathrm{NO}_{2}+2 \mathrm{H}_{2} \mathrm{O}+\mathrm{O}_{2},
$$

is capable of oxidizing the carbon materials surfaces [8]. The gas mixture components can be arranged in the following order on the basis of the oxidative activity index: $\mathrm{NO}_{2}>\mathrm{N}_{2} \mathrm{O} \approx \mathrm{NO} \approx \mathrm{O}_{2}$.

It was shown in [9-10] that when nitrogen dioxide is reduced, nitric oxide (II) is predominantly formed. The absence of this component in the composition of gaseous products of CNT oxidation can be explained by the fact that NO is rapidly oxidized by oxygen formed by reaction (1). The absence of molecular nitrogen in products of the interaction of carbon with nitrogen oxides is indicated in [11]. In [12] it is noted that in addition to NO in the products of oxidation of carbon materials with nitrogen dioxide, $\mathrm{N}_{2} \mathrm{O}$ is identified.

Thus, the information obtained on the composition of gaseous products of CNT oxidation in nitric acid vapor does not contradict the literature data. The chemistry of the oxidation of the CNT surface with the nitric acid thermal decomposition products can be described by a set of typical reactions, the most important of which are the 
processes of formation of oxygen-containing functional groups, processes of formation and transformation of nitrogen-containing surface formations, processes of elimination of oxygen-containing functional groups, processes of amorphous phase oxidation, and associated processes of interaction between reaction products. Water vapor present in the oxidizing mixture catalyzes the oxidation without changing the interaction mechanisms [13].

Nitric oxide (I) is identified in gaseous products $\sim 2.5$ $\mathrm{h}$ after the oxidizing reagent supply started in fairly small amounts (up to 1.7 v.\%). The maximum $\mathrm{N}_{2} \mathrm{O}$ concentration is observed in oxidation products purified from admixtures of the CNT metal oxide catalyst, the degree of functionalization of which using the oxygencontaining groups is lower than in the untreated CNTs subjected to the similar treatment. On this basis, it can be concluded that the catalyst particles, exerting an accelerating effect on the creation of functional groups, contribute to a decrease in the rates of the $\mathrm{N}_{2} \mathrm{O}$ formation reactions.

The total volumes of gases released at equal intervals are determined by the rate of the oxidizing reagent supply. The predominant component of the analyzed mixtures of gaseous products is $\mathrm{NO}_{2}$. Its concentration practically does not change during the process. It ranges from 71.2 to $81.5 \mathrm{v} . \%$. The consistency of the $\mathrm{NO}_{2}$ content is due to its excessive amount in the reaction mass and a low degree of conversion.

The obtained data were used to estimate the total thermal effect and heating rate of the reaction mass due to that effect (Table 2). For the oxidation of the CNTs containing the metal oxide catalyst admixtures, the total amount of the reaction heat released per unit time is 1.8 to 5 times higher than in the case of the oxidation of the purified CNTs. This fact lies in accordance with the assumption of the catalytic influence of the metal oxide inclusions on the process.

Table 2. Calculated values of the total amount of reaction heat $(q r)$ and superheating of the reagents without considering thermal losses $(d t)$ during the oxidation of $1 \mathrm{~g}$ of the CNTs in concentrated nitric acid vapors at $140^{\circ} \mathrm{C}$

\begin{tabular}{|l|l|l|l|}
\hline CNT type & Time, $\mathrm{h}$ & $q r, \mathrm{~J}$ & $d t, \mathrm{~K}$ \\
\hline \multirow{5}{*}{ initial } & 1.5 & 15.07 & 3.01 \\
\cline { 2 - 4 } & 2.5 & 32.45 & 9.21 \\
\cline { 2 - 4 } & 3.5 & 26.37 & 7.52 \\
\cline { 2 - 4 } & 4.5 & 24.41 & 6.98 \\
\cline { 2 - 4 } & 6.0 & 19.54 & 4.08 \\
\hline \multirow{5}{*}{ purified } & 1.5 & 8.62 & 3.77 \\
\cline { 2 - 4 } & 2.5 & 6.45 & 6.20 \\
\cline { 2 - 4 } & 4.5 & 6.18 & 5.97 \\
\cline { 2 - 4 } & 6.0 & 4.96 & 3.51 \\
\hline
\end{tabular}

\section{Modeling of the Temperature Field of the Reaction Zone Inside the CNT Gas- Phase Oxidation Reactor}

The purpose of thermal calculation is to determine the geometric characteristics of the reaction region (diameter and height) that provide an allowable volumetric temperature drop in the CNT bulk layer during the gasphase functionalization.

The temperature field in the CNT bulk layer is modeled through solving the non-stationary heat conduction problem for a finite cylinder with a volumetric heat source.

Since the gas flow rate in the layer does not exceed 0.3 $\mathrm{mm} / \mathrm{s}$, the gas medium is assumed to be stationary, which provides an additional margin for the temperature difference.

The temperature of the medium from the lateral cylinder surface may be used as a reference point, thereby making it possible to implement homogeneous boundary conditions on this surface (Fig. 4).

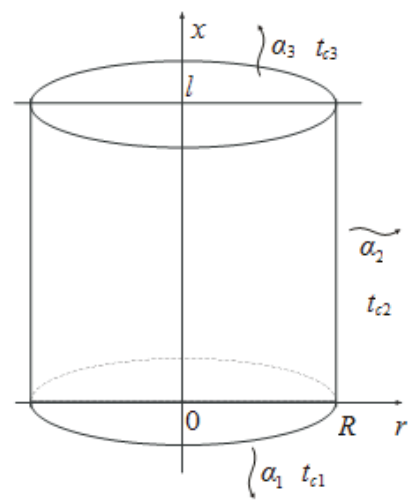

Fig. 4. A finite cylinder

The problem has the following form:

$$
\begin{gathered}
\frac{\partial t(x, r, \tau)}{\partial \tau}=a\left(\frac{\partial^{2} t(x, r, \tau)}{\partial x^{2}}+\frac{\partial^{2} t(x, r, \tau)}{\partial r^{2}}+\frac{1}{r} \frac{\partial t(x, r, \tau)}{\partial r}\right)+ \\
+\frac{q v}{c \rho}, \quad 0<x<l ; \quad 0<r<R \\
t(x, r, 0)=f(x, r)-t_{c 2} \\
\quad \frac{\partial t(x, 0, \tau)}{\partial r}=0 \\
\lambda \frac{\partial t(0, r, \tau)}{\partial x}-\alpha_{1}\left(t(0, r, \tau)-t_{c 1}+t_{c 2}\right)=0 \\
\lambda \frac{\partial t(l, r, \tau)}{\partial x}+\alpha_{3}\left(t(l, r, \tau)-t_{c 3}+t_{c 2}\right)=0 \\
\lambda \frac{\partial t(x, R, \tau)}{\partial r}+\alpha_{2} t(x, R, \tau)=0
\end{gathered}
$$

Here, $t(x, r, \tau)$ is the temperature field of a bounded cylinder as a function of the longitudinal coordinate $x$, the radial coordinate $r$, and the time $\tau ; q_{v}$ is the specific thermal power of a volumetric heat source, $\mathrm{J} / \mathrm{m}^{3} ; \lambda, c, \rho$ respectively, thermal conductivity, $\mathrm{W} /(\mathrm{m} \cdot \mathrm{K})$, heat capacity, $\mathrm{J} /(\mathrm{kg} \cdot \mathrm{K})$, density of the cylinder material, $\mathrm{kg} / \mathrm{m}^{3}$; $f(x, r)$ is the initial temperature distribution; $\alpha_{1}, \alpha_{2}, \alpha_{3}$ 
heat transfer coefficients from the outer surfaces of the cylinder, $\mathrm{W} /\left(\mathrm{m}^{2} \cdot \mathrm{K}\right) ; t_{c 1}, t_{c 2}, t_{c 3}$ - ambient temperatures from the side of the corresponding surfaces; $R, l$ - the radius and height of the cylinder, $\mathrm{m}$, respectively.

The solution of problem (2) - (7), obtained by the method of finite integral transformations, has the following form:

$$
\begin{gathered}
t(x, r, \tau)=t_{c 2}+\sum_{n=1}^{\infty} \sum_{m=1}^{\infty} \frac{1}{N_{m n}} \\
\left(\frac{S+\frac{Q}{a}}{\mu_{m n}}+\left(F-\frac{S+\frac{Q}{a}}{\mu_{m n}}\right) \exp \left(-\mu_{m n}^{2} a \tau\right)\right) . \\
\cdot \sin \left(v_{n} x+\varphi_{n}\right) J_{0}\left(\gamma_{m} r\right)
\end{gathered}
$$

where

$$
\begin{gathered}
F=\int_{0}^{l} \int_{0}^{R}\left(f(x, r)-t_{c 2}\right) \sin (v x+\varphi) r J_{0}(\gamma r) d r d x, \\
S=\frac{R}{\gamma} J_{1}(\gamma R)\left(\begin{array}{c}
\left.\frac{\alpha_{3}}{\lambda}\left(t_{c 3}-t_{c 2}\right) \sin (v l+\varphi)-\right) \\
-\frac{\alpha_{1}}{\lambda}\left(t_{c 1}-t_{c 2}\right) \sin (\varphi)
\end{array}\right) \\
Q=\int_{0}^{l} \int_{0}^{R} \frac{q v}{c \rho} \sin (v x+\varphi) r J_{0}(\gamma r) d r d x= \\
=\frac{q_{v} R}{c \rho v_{n} \gamma_{m}} \cdot\left(\cos \left(\varphi_{n}\right)-\cos \left(v_{n} l+\varphi_{n}\right)\right) J_{1}\left(\gamma_{m} R\right), \\
\varphi=\operatorname{arctg}\left(\frac{\lambda v}{\alpha_{1}}\right)
\end{gathered}
$$

$v$ - successive positive roots of the equation,

$$
\operatorname{tg}(v l+\varphi)=-\frac{\lambda v}{\alpha_{3}},
$$

$\gamma$ - successive positive roots of the equation,

$$
\frac{\lambda \gamma}{\alpha_{2}} J_{1}(\gamma R)-J_{0}(\gamma R)=0,
$$

$J_{1}(r), J_{0}(r)-$ Bessel functions,

$$
\begin{gathered}
\mu_{m n}^{2}=\gamma_{m}^{2}+v_{n}^{2} \\
N_{m n}=\frac{R^{2}}{4}\left(J_{1}^{2}\left(\gamma_{m} R\right)+J_{0}^{2}\left(\gamma_{m} r\right)\right) \\
\left(l+\frac{1}{v_{n}}\left(\begin{array}{l}
\sin \left(\varphi_{n}\right) \cos \left(\varphi_{n}\right)- \\
-\sin \left(v_{n} x+\varphi_{n}\right) \cos \left(v_{n} x+\varphi_{n}\right)
\end{array}\right)\right) .
\end{gathered}
$$

The solution to this problem was used to determine the design and mode parameters of the gas-phase functionalization equipment, at which the maximum temperature difference considering the volume of the CNT bulk layer during the process does not exceed $10 \mathrm{~K}$.

The proposed approaches will give an opportunity to carry out modeling of processes occurring at the CNT oxidation using ozone, hydrogen peroxide vapors [14], and at secondary treatment of carboxylated CNTs using gaseous ammonia to form amide groups.

\section{Conclusions}

The particles of the transition metal oxide admixtures catalyze the formation of carboxyl groups on the CNT surface during the oxidation in nitric acid vapors and contribute to 1.8-5.0 increases in the total thermal effect. The technique of thermal calculation of equipment parameters for performing the process of CNT gas-phase oxidative functionalization is proposed. The data of the experimental studies were used to solve the equations of the mathematical model of the non-stationary temperature field in the working region inside the reactor where the process takes place.

The research was supported by the Russian Science Foundation (Grant No. 15-13-10038).

\section{References}

1. A.V. Melezhyk, A. V. Rukhov, E. N. Tugolukov, A.G. Tkachev, Nanosystems: Phys. Chem. Math. 4, 247 (2013)

2. A. A. Aladinskiy, A. V. Rukhov, E. N. Tugolukov, T. P. Dyachkova, Transactions TSTU. 20, 572 (2014)

3. C. Kingston, R. Zepp, A. Andrady, D. Boverhof et al., Carbon. 68, 33 (2014)

4. Yu.I. Merkulova, S.V. Kondrashov, T.P. Dyachkova, P.S. Marakhovskii, G.Yu. Yurkov, Russ. J. Appl. Chem. 88, 1848 (2015)

5. H.M. Kim, K. Kim, S.J. Lee, J. Joo et al., Curr. Appl. Phys. 4, 577 (2004)

6. J. Yang, X. Li, C. Liu, G. Ma, Appl. Surf. Sci. 325, 235 (2015)

7. H.P. Boehm, Carbon. 40, 145 (2002)

8. B.R. Stanmore, V. Tschamber, J.-F. Brilhac, Fuel. 87, 131 (2008)

9. A. Setiabudi, M. Makkee, J.A. Moulijn, Appl. Catal. B Envir. 50, 185 (2004)

10. Z. Zhang, J.D. Atkinson, B. Jiang, M.J. Root, Z. Yan, Appl. Catal. B Envir. 148-149, 573 (2014)

11. A.R. Chugtai, M.M. Atteya, J. Kim, B.K. Konowalchuk, D.M. Smith, Carbon. 36, 1573 (1998)

12. U. Kirchner, V. Scheer, R. Vogt, J. Phys. Chem. A. 104, 8908 (2000).

13. M. Jeguirim, V. Tschamber, J.F. Brilhac, P. Ehrburger, Fuel. 84, 1949 (2005)

14. T.P. Dyachkova, Yu.A. Khan, N.V. Orlova, S.V. Kondrashov, Transactions TSTU. 22, 232 (2016) 